Aus der Akademie für praktische Medizin in Köln (I. chirurgische Abteilung: Chefarzt: Geh. Med.-Rat Prof. Dr. B a r d e n he u er).

\title{
Über einige seltenere Frakturen und Luxationen des Oberarmkopfes bzw. über die Kombination beider Verletzungsarten.
}

Von Dr. H. Luxembourg, Sekundärarzt der chir. Abteilung des Bürgerhospitals.

(Mit 1 I Abbildungen.)

In folgendem möchte ich zunächst über 4 Fälle von gleichzeitiger Fraktur und Luxation des Oberarmkopfes berichten, die im Laufe der letzten 8 Jahre im Kölner Bürgerhospital zur Behandlung kamen, und die in mehrfacher Hinsicht von Interesse sind. Die Zahl ähnlicher bisher veröffentlichter Fälle ist eine verhältnismäßig geringe. $\mathrm{Mas}$ on ${ }^{1}$ ), der einen Fall von doppelseitiger Schulterverrenkung sah, in dem rechts außer der Luxation der Kopf im chirurgischen Halse abgebrochen war, stellte mit dem von ihm beobachteten insgesamt 64 Fälle aus der Literatur zusammen; als die seiner Ansicht nach beste Methode wandte er bei seinem Patienten folgendes Behandlungsverfahren an: Bei peinlichster Asepsis wurde nach Eröffnung des vorderen Abschnittes des Schultergelenkes der frakturierte Kopf mittels eines Elevatoriums an seine richtige Stelle gebracht, da auf eine andere Weise eine Einrenkung nicht gelingen wollte. Das Gelenk wurde 4 Wochen ruhig gestellt, und auf diese Art ein sehr gutes funktionelles Resultat erzielt.

Von den 64 Fällen Mas ons wurden 23 mit Arthrotomie behandelt, davon $\mathbf{1} 4=70$ Proz. mit gutem Erfolg, 2 I wurden reseziert, davon $9=42$ Proz. mit gutem Resultat. Auch

I) The treatement of dislocation of the shoulder-joint complicated by fracture of the upper extremity of th humerus. Annals of surgery 1908, Mai. Ref. im Zentralbl. f. Chir. I908, Bd. 35. 
F a cque ${ }^{1}$ ) empfiehlt auf Grund von 38 bisher publizierten Beobachtungen die Resektion des Kopfes als die rationellste Methode, da hierdurch die Beweglichkeit des Armes meist eine in jeder Hinsicht zufriedenstellende werde, während durch die konservative Behandlung entweder totale Ankylose oder jedenfalls eine bedeutende Beschränkung der Beweglichkeit zustande komme; von den 38 Fällen $\mathrm{F}$ a cques betrafen 22 solche Fälle, die längere Zeit verfolgt werden konnten, und bei welchen ein ausgezeichnetes Ergebnis der Operation festzustellen war. Auch bei unseren Fällen interessierte uns vorzugsweise das Dauerresultat der von uns benutzten blutigen und unblutigen Methoden, und habe ich zur Feststellung desselben die einzelnen Fälle, soweit es mir möglich, in der letzten Zeit nachuntersucht. Das Ergebnis der Nachuntersuchung ist den einzelnen Krankengeschichten beigefügt.

$\mathrm{DaB}$ die konservative unblutige Behandlung hinsichtlich des Erfolges manches zu wünschen übrig läßt, zeigt auch der von uns im Jahre 1903 beobachtete Fall, den wir, von einem operativen Eingriff Abstand nehmend, lediglich der Extensionsbehandlung unterzogen, und dessen Krankengeschichte zunächst folgt.

Fall I. K. H., 35 Jahre alt, Nachtwächter, aus Köln. Aufgenommen 2. IV. 1903, entlassen 22. V. I903.

Diagnose: Fract. humeri dextr. pertubercularis et tuberculi maj. cum luxatione capitis hum. praeglenoidal:

Nachts in eine $2 \mathrm{~m}$ tiefe Luke gefallen, dabei auf die rechte Schulter aufgeschlagen; Schmerzen in der rechten Schulter und Gebrauchsunfähigkeit des rechten Armes.

A uf $\mathrm{n}$ ahmebef und: Sehr starke Schwellung (Querdurch. messer rechts 18 , links $10,5 \mathrm{~cm}$ ) und starker Druckschmerz am rechten Schultergelenk; ausgedehnte Hautblutung, von der Mitte des Oberarmes beginnend, nach hinten bis zum Schulterblatt und auf der Brust bis zum Sternum reichend; Pulsation auf der Höhe der Schwellung nicht zu fühlen, auch daselbst kein Geräusch zu hören; Radialispuls ist kräftig, regelmäßig. Von der Axilla aus ist der Humeruskopf nicht tastbar; dagegen ist unter dem vorderen $\mathrm{Ab}$ schnitt des M. deltoideus eine knöcherne Resistenz, wahrscheinlich

I) Du traitement de certaines fractures graves de l'extremité supérieure de l'humerus par la résection de la tête hnmérale. Thèse de Paris I909. Ref. im Zentralbl. f. Chir. roro, Bd. 37. 
der Kopf, fühlbar; bei Bewegungen des nicht federnd fixierten und scheinbar auch nicht verkürzten Oberarmes Krepitation im Schultergelenk.

Röntgenogramm (Fig. 1) zeigt einen Bruch des Oberarmkopfes in der Höhe der Tubercula mit Luxation desselben vor die Gelenkpfanne; zwischen letzterer und Oberarmschaftende liegt das ebenfalls abgesprengte Tuberculum majus.

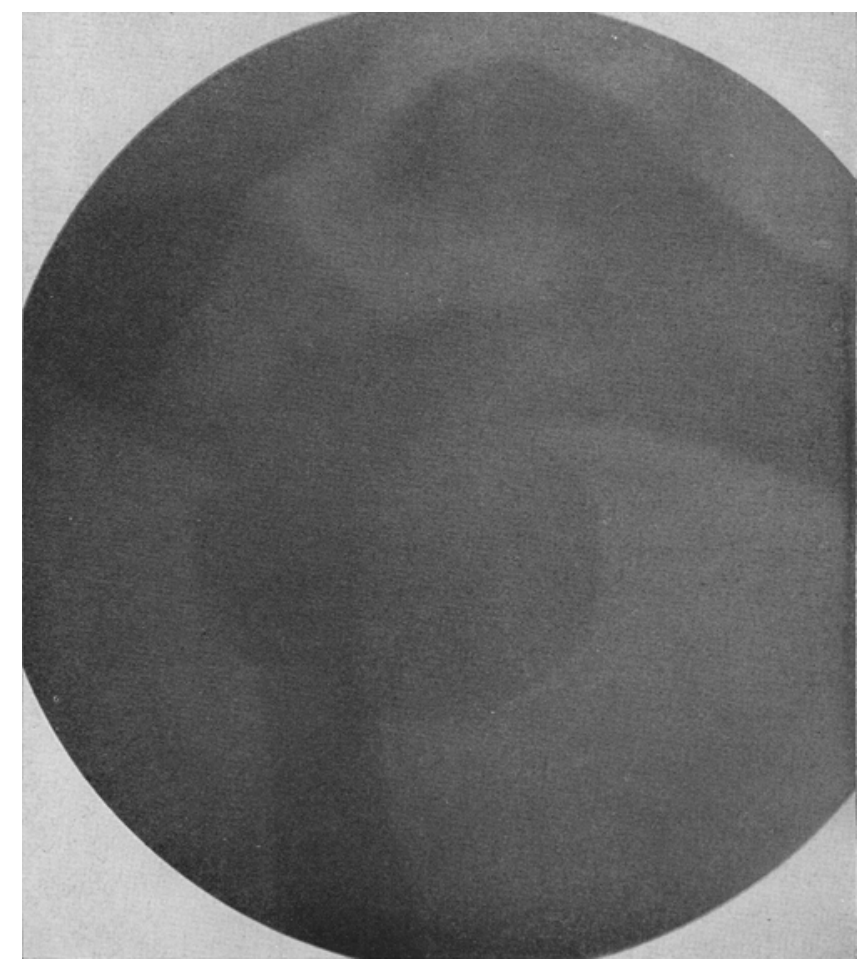

Fig. I.

B ehandlung: Längsextension im Bette fußwärts mit $6 \mathrm{~kg}$ Belastung nach vergeblichem Versuche, in Narkose den Kopf zu reponieren. 6. IV. Längsextension in mäßiger Abduktion im Winkel von $45 \mathrm{Grad}$ mit gleicher Belastung. 7. IV. Dieselbe Extension in Abduktion von 9o Grad. 8. IV. Gleiche Extension nach hinten oben außen. 23. IV. Patient seit einigen Tagen außer Bett; Massage; aktiv-passive Übungstherapie, insbesondere Stabübungen. Der Arm kann aktiv noch vorn und seitlich bis zur Horizontalen gehoben werden. 
Das Röntgenogramm zeigt keine Veränderung gegenüber dem Röntgenbefunde bei der Aufnahme, abgesehen von mäßigem periostalen Callus am oberen Ende des Humerusschaftes.

22. V. Zur ambulanten Behandlung der Poliklinik entlassen. Rechtes Schultergelenk vorn etwas stärker gewölbt als das linke und im Tiefendurchmesser verbreitert; aktiv wird der Arm seitlich und nach vorne nur wenige Grade über die Horizontale gehoben; Außen- und Innendrehnug ist etwa $1 / 3$ und Heben des Armes nach hinten nur ca. 20 Grad möglich.

Nachuntersuchung: Nicht möglich, da K. nach Auskunft der Verwandten am 27. VII. 1904 an Lungenschwindsucht gestorben ist.

Von besonderer Wichtigkeit für die Bewertung der Behandlungsmethode war in diesem Falle der bei Beendigung des Ex. tensionsverfahrens erhobene röntgenologische Befund, insofern nach diesem eine Beeinflussung der Bruchstuicke, insbesondere des verrenkten Oberarmkopfes hinsichtlich seiner Lage völlig auszuschließen war.

Demgemäß entschlossen wir uns I 906 für einen weiteren Fall zum operativen Eingriff, der aber leider nicht den gewünschten Erfolg hatte, und bei dem es während der Nachbehandlung nach anfänglich günstigem, fieberfreiem Heilungsverlaufe zu septischen Prozessen kam, die schließlich eine Exartikulation des Armes erforderlich machten. Gerade unter besonderer Berücksichtigung dieses Falles schließen wir uns der Forderung Masons einer peinlichsten Asepsis bei derartigen Operationen in jeder Hinsicht, an, weiter auch dieselbe als dringend notwendig erachtend bei der Nachbehandlung, zumal wenn eine reichliche Blutansammlung im Schultergelenke oder ausgedehnte Zerreißung der Gelenkkapsel unld Zerquetschung der umgebenden Weichteile, der Muskulatur und der Haut, eine Gelenkdrainage als zweckmäßig erscheinen lassen. Speziell der Quetschung der Weichteile wegen empfiehlt daher F a cq u e auch die Operation nicht zu frühzeitig vorzunehmen, da sonst das Resultat derselben gefährdet werde, und ist seiner Meinung nach, der wir auf Grund weiterer Beobachtungen beipflichten, der beste Zeitpunkt zur Vornahme der Resektion zwischen dem 7. und ı. Tage nach der Verletzung, da man später bei längerem Warten mit beginnender Atrophie der Muskeln zu rechnen hat. 
Fall 2. S. A., 33 Jahre alt, Installateur, aus Köln. Aufgenommen 4. XI. 1906, entlassen 23. I. 1907.

Diagnose: Fract. humeri dextr. pertubercularis cum luxatione capitis infraglenoidal.

Beim Ausweichen vor der elektrischen Straßenbahn vom Rade gestürzt, dabei von einem Kraftwagen angefahren und vom Vorderrade des letzteren mit der rechten Schulter über den Boden ge-

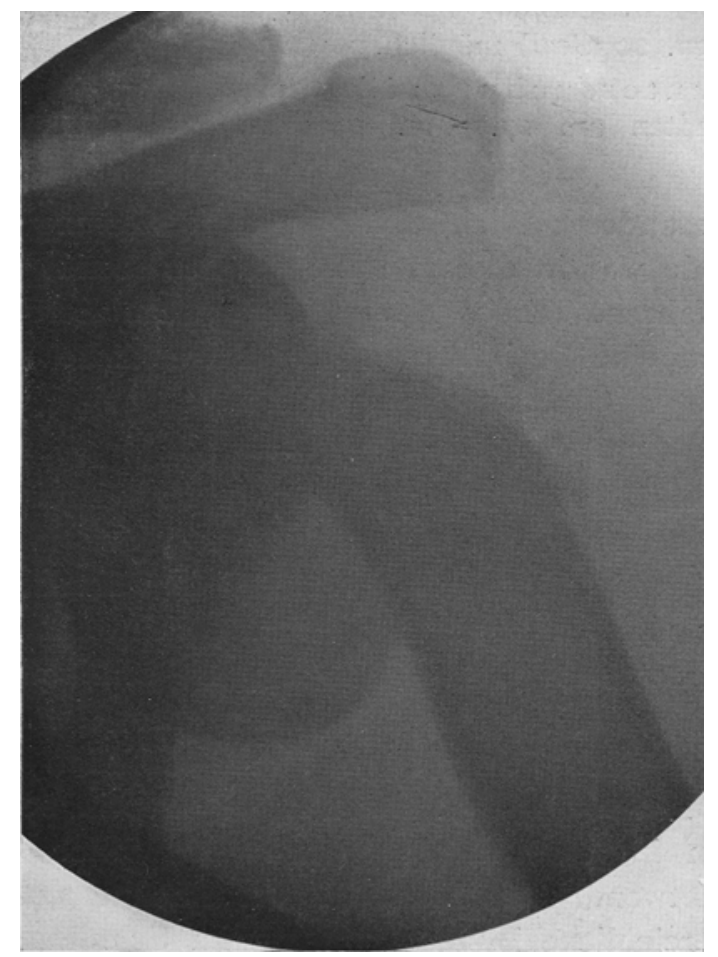

Fig. 2.

schleift; heftige Schmerzen in der rechten Schulter und Gebrauchsunfähigkeit des rechten Armes.

A uf nahmebefund: Sehr starke Schwcllung des rechten Schultergelenks bis zur Oberarmmitte; daselbst großer Druckschmerz; Oberarm steht, nicht federnd fixiert, in geringer seitlicher Abduktion, ist scheinbar etwas verkürzt; bei Bewegungen Krepitation am oberen Humerusende. In der Achselhöhle ist, diese teilweise ausfüllend, eine kugelförmige, knöcherne Resistenz, der Oberarmkopf, fühlbar, der bei Bewegungen des Oberarms unbeweglich in seiner Lage verharrt. Das Röntgenogramm (Fig. 2) zeigt einen Bruch des Humcrus- 
kopfes in der Höhe der Tubercula mit Dislokation des Kopfes unter beziehungsweise vor die Gelenkpfanne des Schulterblattes; das abgeschrägte, obere Humerusschaftende ist mit seiner Innenkante gegen die untere Pfannenhälfte gerichtet.

Behandlung: Velpeauscher Verband. 5. XI. In Narkose Versuch, den Humeruskopf zu reponieren, mißlingt, deshalb 6. XI. in Narkose Arthrotomie (Geheimrat B ard en h e u er) vom O 11 i e r schen Schnitte aus; Exstirpation des Kopfes nach Entfernung von reichlichem, fluissigem und geronnenem Blut aus dem Gelenk; dann Katgut-Gelenkkapsel- und Seidenhautnaht; keine Drainage. Gipsverband bei senkrecht eleviertem Oberarm und rechtwinklig gebeugtem Ellbogengelenk und mit in Pronation auf dem Kopfe fixierter Hand mit BrusteinschluB. - i6. XI. Entfernung des Gipsverbandes und Anlegung eines Extensionsverbandes am elevierten Arme mit $5 \mathrm{~kg}$ Belastung; aus dem unteren Wundwinkel starke Sekretion. 28. XI. Im Laufe der letzten Tage unter Temperatursteigerung mehrere septische Blutungen an der Operationsstelle bei zunehmender Schwäche des Patienten. 3. XII. Wicderum heftige, den Patienten in hohem Maße schädigende, in Narkose nicht zu stillende Nachblutung; deshalb Unterbindung der Art. subclavia in der Mitte nach temporärer Resektion der Clavicula. - - 4. XII. Wegen beginnender Gangrän der Hand und des Vorderarmes und des schlechten Allgemeinzustandes des Patienten Exartikulation des Armes im Schultergelenk mit ausgiebiger Drainage. - I 5. XII. Wundfläche an der Exartikulationsstelle sezerniert ziemlich stark, ist mit frischen Granulationen bedeckt; keine Temperatursteigerung mehr. Patient hat sich gut erholt. - 23. I. 1907. Auf Wunsch mit handtellergroßer Granulationsfläche an der Schulter und bei gutem Allgemeinbefinden zur Ambulanz entlassen.

Nachuntersuchung am 26. XI. I9I I: Narbe an der rechten Schulter von guter Beschaffenheit; akromiales Ende der rechten Clavicula etwas nach oben abgewichen; starke Verdickung der letzteren in der Mitte. S. hat zeitweise noch Schmerzen an der Exartikulationsstelle.

Auch in den beiden folgenden Fällen wurde die Resektion des verrenkten Oberarmkopfes vorgenommen; was die Schnittführung zur Eröffnung des Schultergelenkes betrifft, so kommt nach unseren Erfahrungen nur der Langenbecksche vordere Schnitt als der praktischste in Frage; daß letzterer nach der Exstirpation des Kopfes auch eine vorzügliche Übersicht über die Gelenkhöhle zwecks Entfernung event. in derselben noch vorhandener Knochensplitter und zur ausgiebigen Ausspülung des Gelenkes gestattet, bedarf keiner weiteren Erörterung. 
Fall 3. B. M., 2I Jahre alt, Büfettfräulein, aus Köln. Aufgenommen 26. IX. I9I0, entlassen I5. XI. I9Io.

Diagnose: Fract. humerisin. pertubercularis cum luxatione capitis praeglenoidalis.

Sturz auf der Treppe und dabei Fall auf die linke Schulter. Klagen über heftige Schmerzen in der Schulter und Gebrauchsunfähigkeit des linken Armes.

A uf $\mathrm{n}$ ahmebef und: Starke Schwellung der linken Schulter mit ausgedehnter Hautblutung; Lage des Kopfes infolge der Weichteilschwellung nicht genau feststellbar; Akromion springt mehr vor

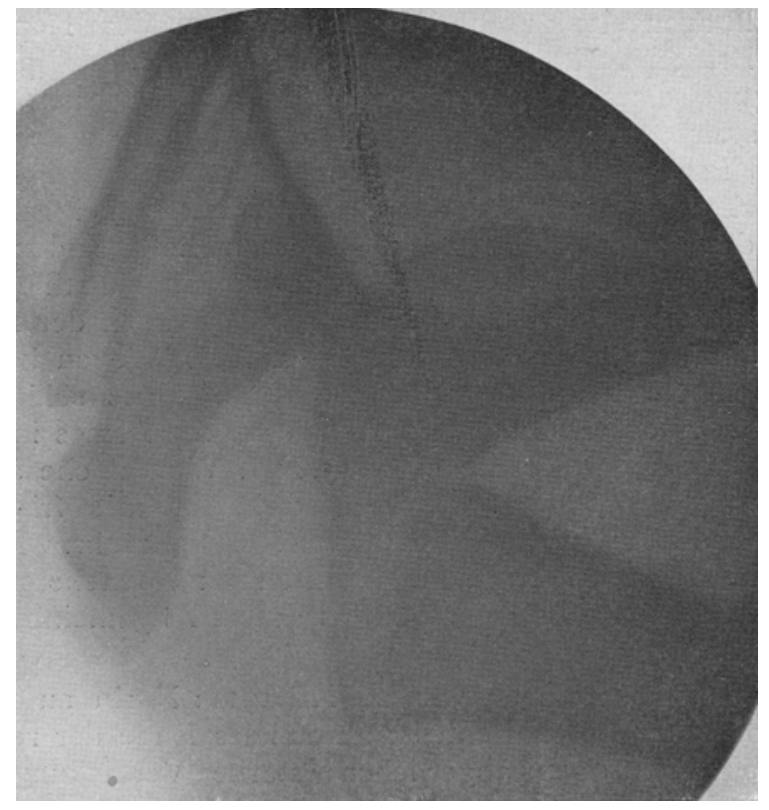

Fig. 3.

als rechts; Schulterblattpfanne nicht deutlich tastbar. Deutliche Krepitation bei Bewegung des Oberarmes in der Höhe des Collum chirurgicum; keine ausgesprochene federnde Fixation des etwas seitlich abduzierten Oberarmes; letzterer scheinbar nicht verkürzt. Ausgedehnte Hautabschürfungen an der Schulter und am. Unterarme. Geringe Behinderung der Beweglichkeit des Ellbogengelenks ohne daselbst nachweisbare Knochenkrepitation. Kräftiger, regelmäßiger Radialispuls; keine nervösen Störungen am Arme.

Röntgenogramm (Fig. 3) zeigt einen Querbruch des Oberarmkopfes in der Höhe der Tubercula mit Luxation desselben vor die 
Gelenkpfanne des Schulterblattes. Das obere Ende des etwas nach außen abweichenden Humerusschaftes steht mit seiner medialen Kante auf dem lateralen Rande des Kopfes. Am Ellbogengelenk keine röntgenologisch feststellbaren Knochenveränderungen.

Beh and lung: Eisunschläge; Velpeauscher Verband. 30. IX. In Narkose Versuch, den Humeruskopf zu reponieren; da dieser mißlingt, sofortige Arthrotomie (Dr. Luxembourg) vom vorderen Langenbeckschen Schnitte aus; Exstirpation des luxierten Kopfes und Glättung des obcren Endes des Humerusschaftes zwecks Einpassung

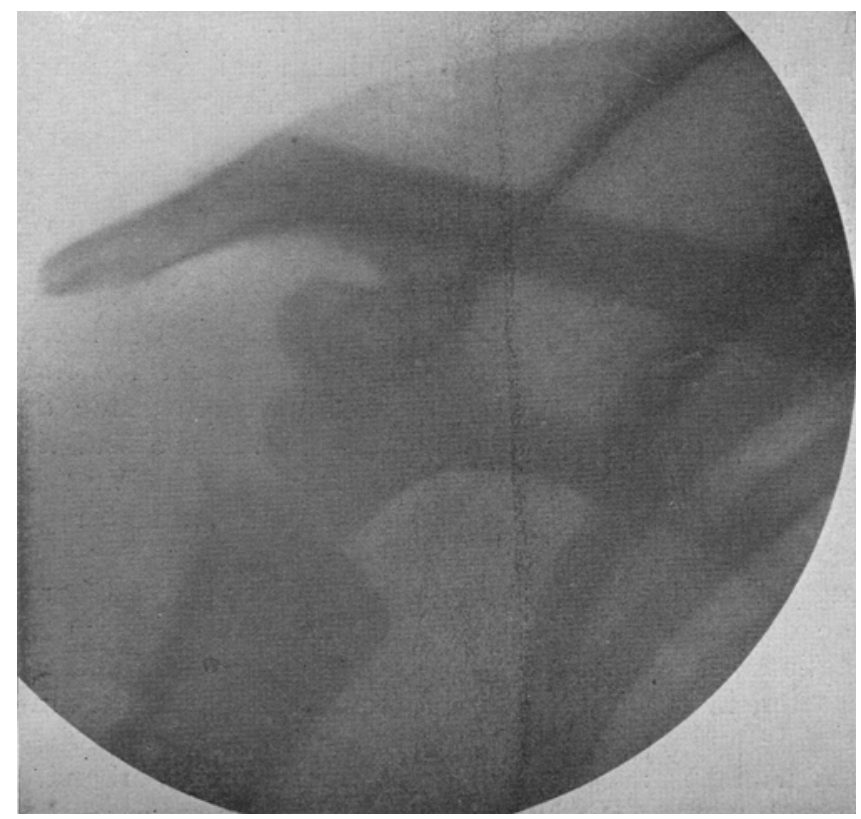

Fig. 4 .

in die Pfanne; Katgutkapsel- und Seidenhautnaht bis auf ein kleines, in den unteren Wundwinkel eingeführtes, Zigarettdrain. Gipsverband bei rechtwinklig seitlich abduziertem Oberarm und rechtwinklig gebeugtem Ellbogengelenk mit Einschluß der Brust. 2. X. Heilungsverlauf ohne Komplikationen; Entfernung des Zigarettdrains. - 7. X. Entferryung des Gipsverbandes; Anlegung eines Extensionsverbandes am rechtwinklig elevierten Arme. - i6. X. Operationswunde vernarbt; aktiv-passive Bewegungsübungen des Ellbogen- und Handgelenks. 3o. X. Patientin verläßt das Bett und macht Stabübungen. 15. XI. Auf Wunsch entlassen. Der linke Arm wird aktiv im Schultergelenk seitlich und nach vorn bis fast zur Vertikalen gehoben; 
Außen- und Innendrehung desselben in geringem Grade behindert. Die übrigen Gelenke des Armes sind frei beweglich; geringe Atrophie der Muskulatur desselben; rohe Kraft kaum herabgesetzt.

Röntgenogramm (Fig. 4) zeigt das obere Ende des IIumerusschaftes unter dem unteren Pfannenrande stehend und im Bereiche der Berührungsstelle beider Knochen, allenthalben vom Periost aus sich neubildende Knochensubstanz.

Nach untersuchung: Nicht möglich, da Patientin von Köln verzogen ist.

Fall 4. D. E., 76 Jahre alt, Tagelöhner, aus Köln. Aufgenommen 19. X. I910, entlassen IO. I. I9II.

Diagnose: Fract. capitis humeri sin. pertubercularisetfract.tuberculi maj. cum luxationecapitis praeglenoid al.

Auf der Straße ausgeglitten und auf die linke Schulter gefallen; starke Schmerzen in der Schulter und Gebrauchsunfähigkeit des linken Armes.

A uf nahmebefund: Ziemlich magerer, dekrepider Mann. Starke Schwellung und große Druckempfindlichkeit des linken Schultergelenks; Oberarm etwas seitlich abduciert, nicht federnd fixiert; bei Bewegungen desselben Krepitation am oberen Ende desselben. Oberarmkopf deutlich fühlbar in der Fossa infraclavicularis; unterhalb des Akromions ist eine deutliche dellenförmige Vertiefung der Weichteilc sicht- und fühlbar. Radialispuls mittclkräftig, regelmäßig; keine nervösen Störungen am Arme.

Röntgenogramm ( $\mathrm{Fig}$. 5) zeigt einen Bruch des Oberarmes in der Höhe der Tubercula mit Aussprengung des Tuberculum majus und Luxation des Kopfes vor die l'fanne; das obere Ende des IIumerusschaftes, insbesondere das Tuberculum minus, steht am unteren Pfannenrande.

Behandlung: Eisumschläge, Velpcauscher Verband. 20. X. In Narkose Versuch, den Ilumeruskopf zu reponieren, mißlingt; deshalb Arthrotonie (Geheimrat $\mathrm{B}$ a r l e $\mathrm{n}$ he u er) vom vorderen Längsschnitte aus; Exstirpation des Kopfes und des isolierten Tuberculum majus; Katgutkapsel- und Seidenhautnaht bis auf ein kleines, in den unteren Wundwinkel eingeführtes Zigarettdrain. (iipsverband bei rechtwinklig seitlich abduziertem Oberarme und rechtwinklig gebeugtem Ellbogengelenk mit Einschluß der Brust.

22. X. Heilungsverlauf ohne Komplikationen, Entfernung des Zigarettdrains. - 28. X. Entfcrnung der Haunähte; geringe Sekretion aus der Drainagestelle. - I4. XI. Entfernung des Gipsverbandes; es besteht noch eine kleine, etwas sezcrnierende Gelenkfistel; Beginn mit passiven Bewegungen des Armes. - 2. XII. Die Fistel hat sich geschlossen, Massage; aktiv-passive Bewegungstherapie. - Io. I. I9II. Auf Wunsch entlassen. Operationswunde 
Über einige seltenere Frakturen und Luxationen des Oberarmkopfes usw. 497

vernarbt; aktive Bewegungen des Schultergelenks fast aufgehoben; passive in sämtlichen Ebenen nur in ganz geringem Grade ohne Schmerzen ausführbar. Ellbogen- und Handgclenk frei beweglich; sämtliche Fingergelenke versteift; beim Versuche, die Hand zur Faust zu schlieBen, beträgt die Entfernung der Kuppe des II.-IV. Fingers von der Hohlhand $2-3$, die des Daumens $4 \mathrm{~cm}$.

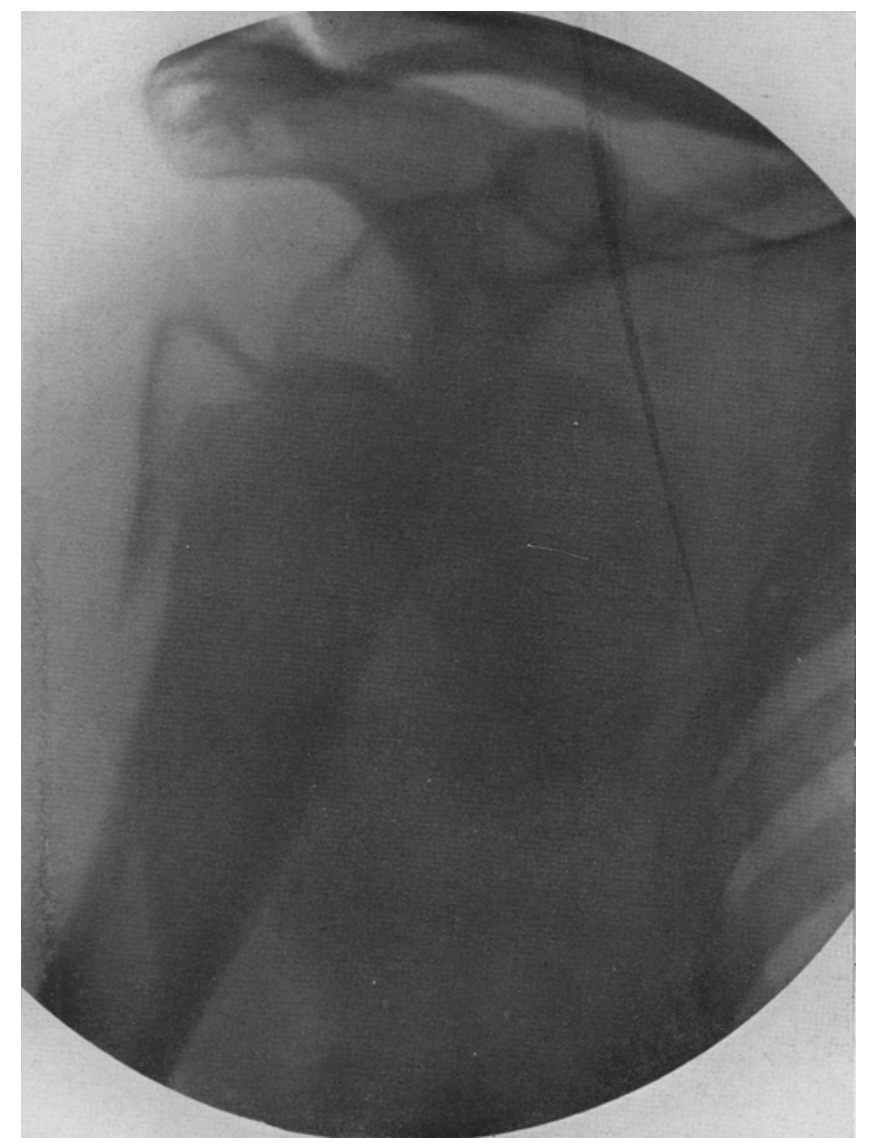

Fig. 5 .

Nachuntersuchung am 5. XI. I9I I: D. ist sehr unbeholfen und nicht fähig, den linken Arm beim An und Auskleiden zu benutzen; cr klagt über zeitweise in der Schulter auftretende heftige Schmerzen; eine Unfalirente bezieht er nicht; im Sommer ist er Verkäufer in einer Mineralwasserbude. Aktiv wird der linke Arm seitlich und nach vorn bis 60 Grad, nach hinten bis 40 Grad gehoben;

Deutsche Zeitschrift f. Chirurgie. I14. Id. 
Außenrotation fast aufgehoben; Innenrotation nur 1/3 möglich; die übrigen Gelenke der linken oberen Extremität sind frei beweglich; geringe Atrophie der Muskulatur derselben; die rohe Kraft derselben ist fast erloschen. Auffallend ist die gute Konfiguration des Schultergelenks, die sich kaum von der der anderen Seite unterscheidet.

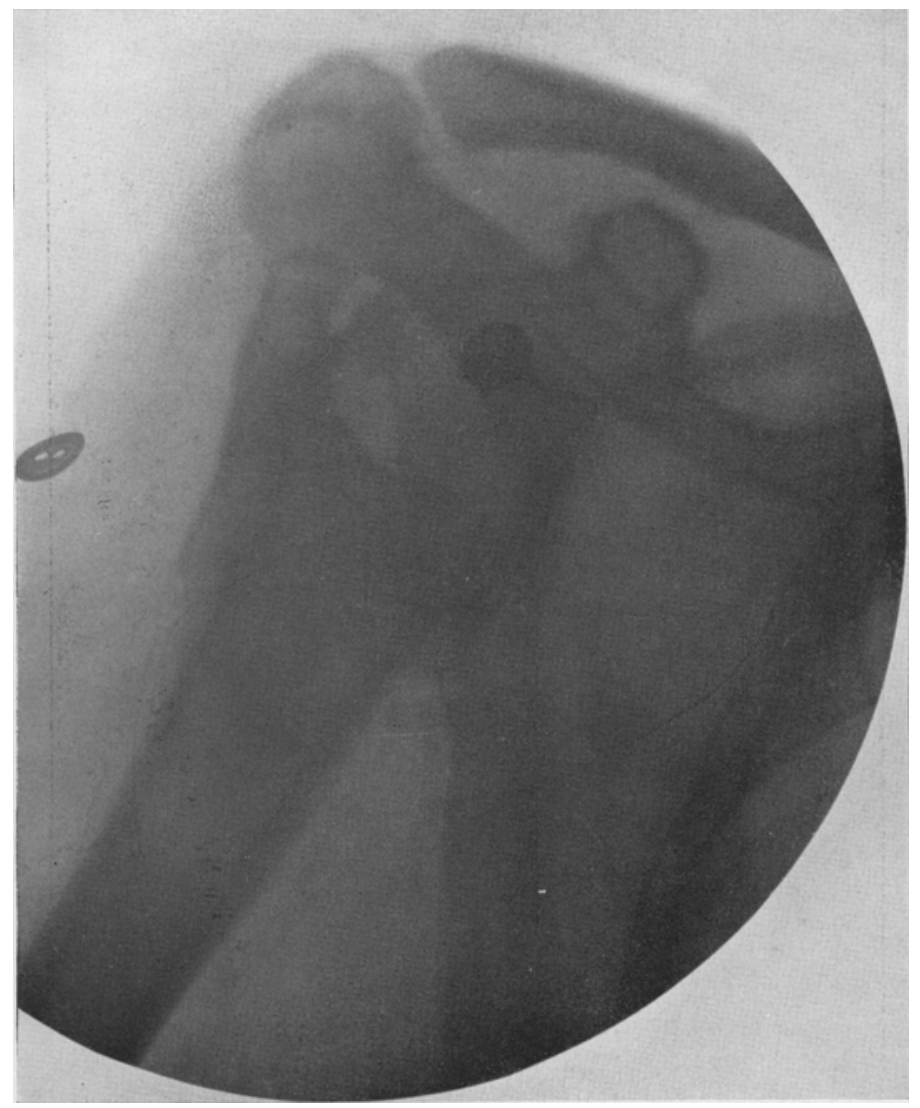

Fig. 6.

Röntgenogramm (Fig. 6) zeigt starke periostale Verdickung des oberen Endes des Humerusschaftes, welcher mit der Schulterblattpfanne, deren Konturen infolge osteoarthritischer Wucherungen fast völlig verwischt sind, und mit dem verdickten Akromion knöchern verwachsen zu sein scheint.

Während im Fall 3 bei der Entlassung der 2 I jährigen $\mathrm{Pa}$ - 
tientin aus dem Krankenhaus die Funktion des Schultergelenkes fast vollständig wiederhergestellt war, trat im Falle 4 bei dem 76 jährigen dekrepiden Manne eine hochgradige Versteifung desselben ein, dic auch trotz der monatelang fortgesetzten Massage und Bewegungstherapie kaum gebessert wurde, was ja weiter bei den chronisch-entzündlichen Knochen- und Knorpelwucherungen der Komponenten des Schultergelenkes nicht befremden kann.

Die 3 folgenden Krankengeschichten betreffen Patienten mit eigentümlichen Bruchformen des Oberarmkopfes, wobei es, ohne daß eine Luxation desselben außerhalb des Gelenkes eingetreten war, zu einer mehr oder weniger großen Verstellung oder gar vollständigen Irehung des Kopffragmentes gekommen war; derartige Fälle pflegen wir gleichfalls operativ in Angriff zu nehmen, da hier das Extensionsverfahren im allgemeinen bei der ausgedehnten Zerreißung des Periosts usw. ohne besonderen Finfluß auf die Stellung des Kopfes bleibt, und letzterer selbst bei sehr großer Belastung des Streckverbandes und starker $\Lambda$ bduktion des Armes unbeweglich in seiner Lage verharrt.

Fall 5. I. G., 63 Jahre alt, Kaufmann, aus Köln. Aufsenommen 9. XI. 1909, entlassen 4. XII. 1909.

J)iagnose: Fract. colli anatomici humeri sin.

Tags zuvor auf der Straße ausgeglitten und mit der linken Schulter auf die Trottoirkante gefallen; Klagen über heftige Schmerzen in der Schulter und Cebrauchsunfähigkeit des linken Armes.

A u f n a h in e b e f und: Mittelgrober, zienlich korpulenter Mann; starke Schwellung und Druckempfindlichkeit des linken Schultergelenks, besonders im vorderen 'Teile; der Oberarmschaft verläuft in normaler Richtung zum Akromion; unter dem vorderen Teile des II. deltoideus eine fühlbare, unbestimmt begrenzte Vorwölbung, wahrscheinlich der Oberarmkopf; bei Bewegungen des Oberarmes Krepitation, scheinbar im Schultergelenk; Oberarm etwas abduziert, nicht rerliüzt.

Rüntgenogramm (Platte nicht mehr vorhanden) zeigt Bruch des Oberarmes im Collum anatomicum und Verstellung des Kopffragmentes derart, daß die Bruchfläche nach innen zur Gelenkfläche der Scapula gerichtet ist.

F e hand lung: Velpeauscher Verband. - II. XI. In Narkose Arthrotomie (Geheimrat Bardenheuer) vom Langenbeckschen Schnitte aus; Exstirpation des Kopfstückes sowie einiger Knochensplitter, 'Trümmer der Tuberculum majus. Katgut-Gelenkkapsel- und 
Seidenhautnaht bis auf ein kleines Glasdrain. Gipsverband bei rechtwinklig seitlich abduziertem Oberarm und rechtwinklig gebeugtem Ellbogengelenk mit Einschluß der Brust. 13. XI. Glatter Heilungsverlauf; Entfernung des Glasdrains. 25. XI. Entfernung des Gipsverbandes. Operationswunde verheilt. Aktiv-passive Übungstherapie, besonders Stabübungen.

26. XI. Röntgenogramm zeigt den Defekt des Oberarmkopfes; das Schaftende hat sich der Pfanne noch nicht genähert. 4. XII. Entlassen mit guter Funktion der Hand-und des Ellbogengelenks und

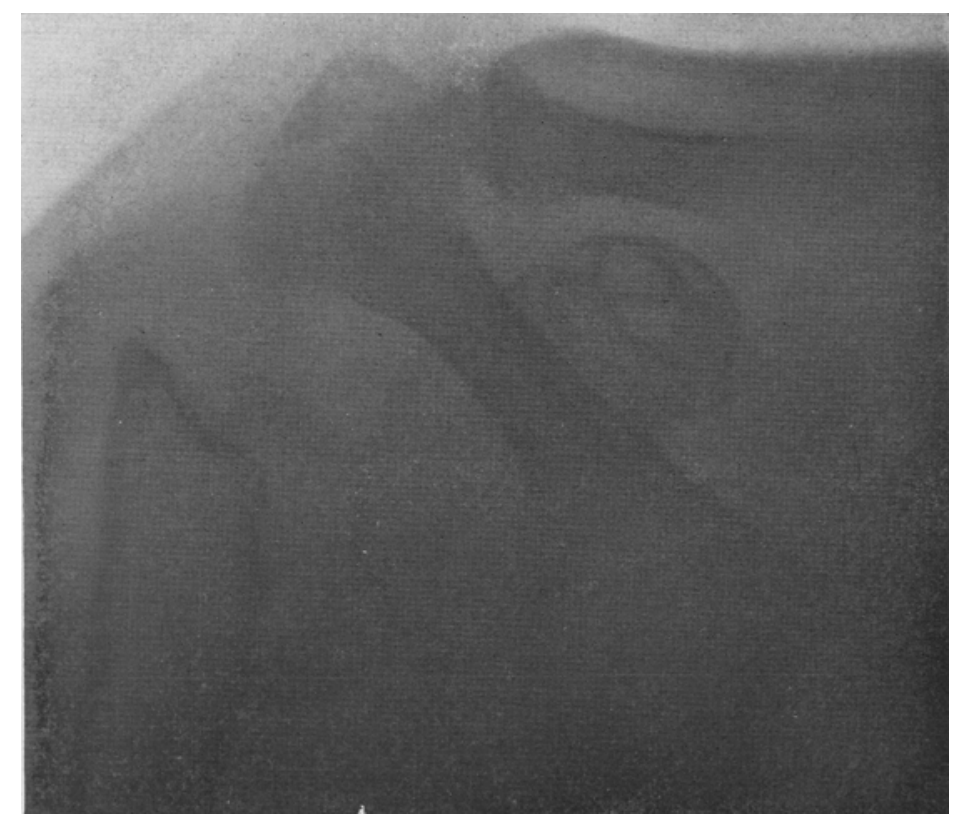

Fig. 7.

schmerzfreier, passiver Beweglichkeit des Schultergelenks bis zur seitlichen Hubhöhe des Armes bis zum rechten Winkel.

$\mathrm{Nachuntersuchung}$ am 5. XI. IgII: L. ist mit der linken Hand äußerst unbeholfen, kann sich nur mit Hilfe anderer an- und auskleiden; bezieht von einer Privat-Unfallversicherung eine Unfallrente von 50 Proz. Aktiv wird der linke Arm nach vorn bis $60 \mathrm{Grad}$, seitlich bis $50 \mathrm{Grad}$, nach hinten bis $30 \mathrm{Grad}$ gehoben; die Außenrotation ist fast aufgehoben, die Innenrotation nur zur Hälfte möglich. Die übrgien Gelenke der linken oberen Extremität sind frei beweglich; die rohe Kraft derselben fast erloschen; hochgradige Atrophie der Schulter, geringere der Armmuskulatur. Auffallend ist die starke Prominenz des Akromions. 
Röntgenogramm (Fig. 7) zeigt mäßige Osteoporose und Osteoarthritis der Komponenten des Schultergelenks, eine beträchtliche Verdickung des Akromions und einen eben sichtbaren Knochenschatten, wahrscheinlich eine zarte knöcherne Verbindung zwischen Oberarmschaft und Schuiterblattpfanne.

F a Il 6. K. A., Frau, 73 Jahre alt, aus Köln. Aufgenommen I. IX. 1910, entlassen r. XI. 1910.

Diagnose: Fract. colli chirurgici humeri dextr.

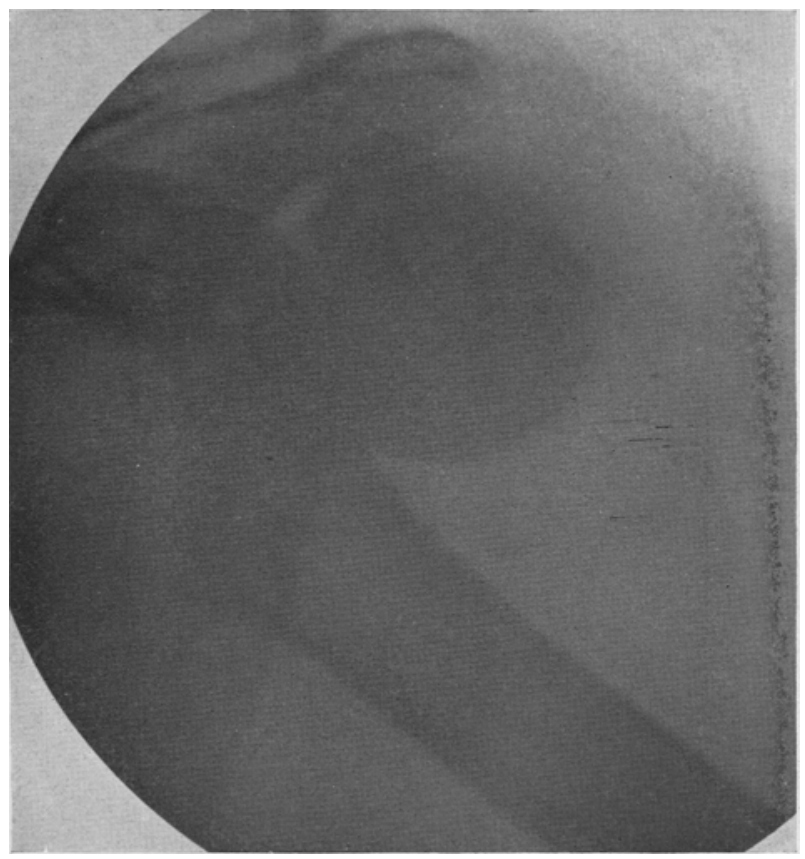

Fig. 8 .

Aus dem Bett auf die rechte Schulter gefallen; Klagen über lieftige Schmerzen in der Schulter und Gebrauchsunfähigkeit des rechten Armes.

A uf nahmebef und: Selır magere, dekrepide Frau; starke Schwellung der rechten Schulter mit Sugillation der Haut; Gegend des Process. coracoid., sowie Oberarmkopf sehr druckschmerzhaft; letzterer außerhalb der Gelenkpfanne, zumal in der Achselhöhle, nicht fühlbar; von hier aus aber deutliches. Krepitieren in der Höhe des Collum chirurgicum fühlbar; keine federnde Fixation des etwas abduzierten Armes, keine Verkürzung desselben.

Röntgenogramm (Fig. 8) zeigt Bruch des Oberarmes im Collum 
chirurgicum und Abweichen des oberen Endes des Oberarmschaftes nach innen; der Kopf ist um I8o Grad gedreht und mit seiner Bruchfläche zum Akromion hin gerichtet.

Behandlung: Längsextension bei rechtwinklig abduziertem Arme, in der Absicht, eine Drehung des Kopfes herbeizufuhren. erfolglos; deshalb 7. IX. in Narkose unblutiger Versuch der Reposition, mißlingt gleichfalls; daher sofortige Arthrotomie (Dr. L u x e m -

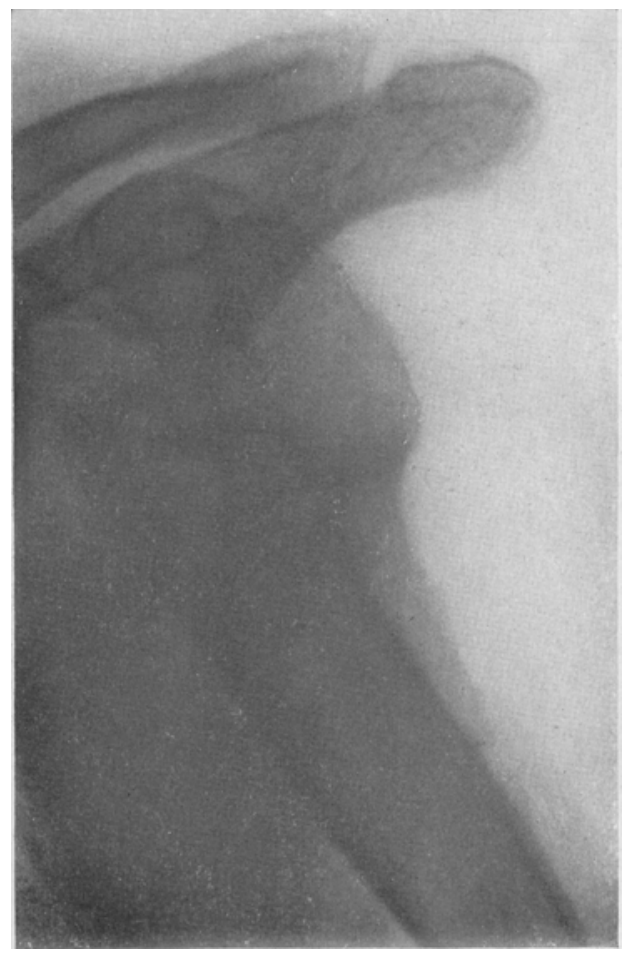

Fig. 9.

b o u r g) vom Langenbeckschen Schnitte aus; Exstirpation des Kopfes, desgleichen zweier größerer isolierter Knochensplitter, Anfrischung des Oberarmschaftendes zwecks Einpassung in die Gelenkpfanne; Katgut-Gelenkkapsel- und Seidenhautnaht. Gipsverband bei rechtwinklig gebeugtem Ellbngengelenk mit Einschluß der Brust.

15. IX. Entfernung des Gipsverbandes; Operationswunde verheilt; Entfernung der Hautnähte; aktiv-passive Ubungstherapie aller Gelenke des rechten Armes. Massage. Faradisation. - I. II. Auf Wunsch entlassen. Operationswunde vernarbt. Alktive Beweglich- 
Uber einige seltenere Frakturen und Inxationen des Oberarmkopfes usw. 503

keit des Schultergelenks in sämtlichen Ebenen im hohen Grade beschränkt; völlige Funktionsfähigkeit der übrigen Gelenke.

Nachuntersuchung am I8. XI. IgIr: Frau $h$ ist im Frühjahr angeblich wegen Darmverschlingung in cinem auswärtigen Krankenhause operiert worden. Aktiv wird der rechte Arm nach vorn und seitlich bis 90 Crad, nach hinten bis 50 Grad gehoben. Die AuBen- und Innenrotation ist nicht behindert. I) ie ubrigen (ielenke des Armes sind frei beweglich; die rohe Kraft ist nicht vermindert.

Röntgenogramm (Fig. 9) zeigt geringe Knochenauflagerung am Akromion, das obere Ende des IIumerusschaftes steht vor der Pfanne.

Was Fall 5 betrifft, so bestimmte uns lediglich die starke Verstellung bzw, die Drehung des Kopffragmentes zur Operation; ist letztere bei ()berarmbrüchen im anatomischen Halse nicht vorhanden, besteht vielmehr nur eine geringe Dislokation des Kopffragmentes oder eine Drehung bis go (irad, so kommt nur die Extensionsbehandlung in Frage; daher kann auch der Vorschlag Buchanans ${ }^{1}$ ), der unlängst 44 Fäle von Brüchen des anatomischen Halses des Oberarmknochens beschrieb, und als die beste Behandlung dieser Fraktur die Exstirpation des abgetrennten Kopfes empfall, da seiner Ansicht nach eine Adaption und künstliche Fixierung desselben am Humerusschafte schwierig sei und ungenügende Resultate liefere, unseren Beifall nicht finden. (aanz besonders war das funktionelle Resultat bei derartigen? Brüchen, die im Laufe der letzten Jahre hier mit Gewichtsextension behandelt wurclen - ihre Zahl ist natürlich bei dem nicht häufigen Vorkommen dieser Verletzung eine beschränkte -., ein in jeder Hinsicht gutes.

So hätte sich auch der folgende Fall vorzüglich zur Extensionsbehandlung geeignet, bei dem eine cigentliche Behardlung der Frakturstelle überhaupt nicht ausgeführt worden ist, und den ich lediglich wegen des interessanten röntgenologischen Isefundes (Figr. Io) beifüge: letzterer demonstriert zur Genüge den Ileilungsprozeß 3 eines nicht behandelten Knochenbruches einer äuBerst indolenten, dem chronischen Alkoholgenuß er-

I) Facture through the anatomical neck of the humerus with dislocation of the head (Annals of surgery igo8, Mai. (Ref. in /entralbl. f. (hir. I9o8, Bd. 35). 
gebenen Patientin; leider fehlt das Resultat der Nachuntersuchung, da die Patientin zu derselben trotz mehrfacher Aufforderung nicht erschien.

F all 7, O. S., Frau, 54 Jahre alt, aus Köln. Aufgenommen 18. III. 1903, entlassen 23. V. 1903.

Diagnose: Fract. capitis humeri sin. pertubercularis vetusta.

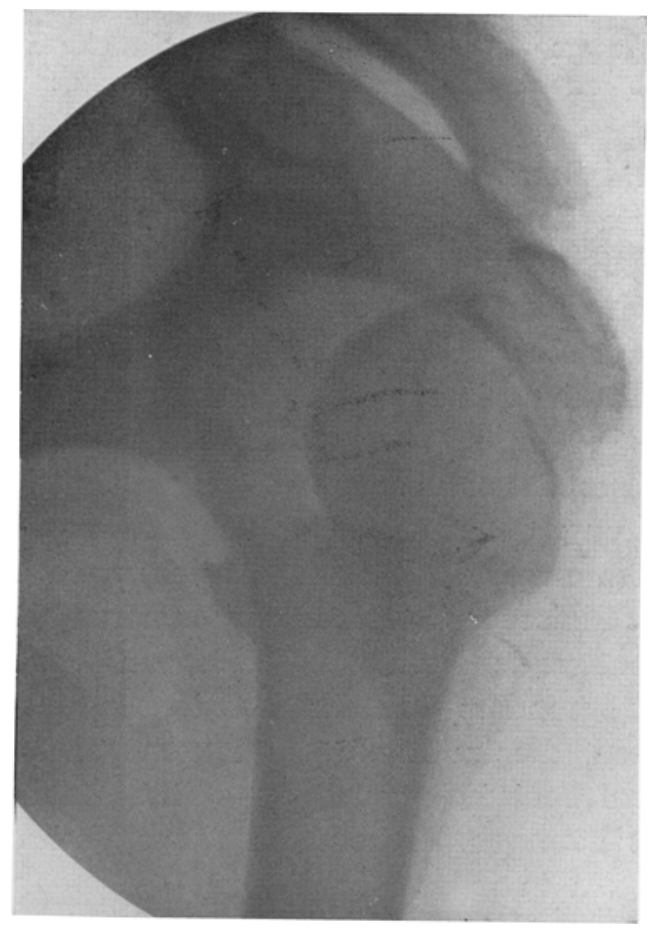

Fig. Io.

Vor 2 Monaten bei Glatteis in der Trunkenheit auf der Straße gefallen und mit der linken Schulter gegen eine Trottoirkante geschlagen; seitdem Schmerzen in der linken Schulter und langsam zunehmende Versteifung des Schultergelenks; bisher ohne ärztliche Behandlung.

Das Röntgenogramm (Fig. Io) zeigt an dem fast völlig in Adduktion verstciften Schultergelenke der im Krankenhause als Säuferin bekannten Patientin eine knöchern verheilte Fractura pertubercularis mit Richtung der Bruchfläche des Kopffragmentes nach außen, lateralwärts, und Interposition des oberen Humerusschaftes zwischen 
Kopffragment und Gelenkpfanne und ausgedehnte osteoarthritische Veränderungen sämtlicher Knochenkomponenten des Schultergelenks.

Aktiv kann der Arm seitlich nicht, passiv bis Schulterhöhe gehoben werden.

Die Behandlung bestand in Massage, Heißluft-Applikation und mediko-mechanischer Therapie. 23. V. Entlassen: Der linke Arm kann aktiv seitlich und nach bis $60 \mathrm{Grad}$ gehoben werden; Innenund AuBenrotation etwa zur Hälfte möglich.

Zum Schlusse lasse ich noch 3 Krankengeschichten von Patienten mit Luxation des Oberarmkopfes folgen, wo letztere teils wegen anderweitiger Verletzungen oder aus sonstigen Gründen vom behandelnden Arzte als solche anfänglich nicht erkannt wurde, und die Reposition des verrenkten Oberarmkopfes längere Zeit unterblieb; im Falle 8 mußte zur Reposition die Arthrotomie vorgenommen werden, da die unblutigen Versuche nicht zum Ziele führten, während diese in den Fällen 9 und to ohne besondere Schwierigkeit gelangen.

Fall 8. C. G., 6o Jahre alt, Fuhrmann, aus Köln. Aufgenommen 21. V. 1909, entlassen 23. VII. 1909.

Diagnose: Luxatio humeri dextr. subcoracoidea et fract. tubercul. maj. vetusta.

Vor 7 Wochen von seinem Lastfuhrwerk beim Scheuwerden des Pferdes über den rechten Oberarm gefahren und dabei einen supracondylären Bruch des letzteren davongetragen. Die zugleich vorhandene Verrenkung des Oberarmkoptes wurde vom behandelnden Arzte nicht erkannt, entsprechende Repositionsversuche daher nicht gemacht und nur der Obcrarmbruch mit Extensionsverbänden behandelt. Klagen über völlige Gebrauchsunfähigkeit des rechten Armes.

A uf nahmebefund: Mittelgroßer, ziemlich magerer Mann; rechter Arm im Schultergelenk in geringer scitlicher Abduktionsstellung fast völlig versteift. Bruchstelle am unteren Oberarmende im Bereiche der Condylen mit mäßiger Verdickung der letzteren und teilweiser Versteifung im Ellbogengelenk verheilt.

Röntgenogramm (Platte nicht mehr vorhanden) zeigt Luxation des Oberarmkopfes vor die Pfanne mit Bruch des Tuberculum majus und verheiltem supracondylären Bruch des Oberarmes.

22. V. Versuche, in Narkose den Kopf zu reponieren, mißlingen. - 24. V. In Narkose Arthrotomic (Geheimrat B ard e n h e u e r) vom hinteren Bogenschnitte aus; mittels Elevatoriums Reposition des Kopfes, keine Drainage; Katgut-Gelenkkapscl- und Seidenhautnaht. Gipsverband bei rechtwinklig abduziertem Oberarm und rechtwinklig gebeugtem Ellbogengelenk mit Brusteinschluß. I. VI. Glatter Hei- 
lungsverlauf; Entfernung der Nähte. I5. VI. Entfernung des Gipsverbandes; aktive und passive Übungstherapie. 25. VI. Massage; Stabübungen.

23. VII. Auf Wunsch entlassen. Operationswunde vernarbt; aktive Beweglichkeit des Schultergelenks nur andeutungsweise vorhanden; Beugung und Streckung im Ellbogengelenk nur zur Hälfte möglich; starke Behinderung der Außenrotation des Vorderarmes.

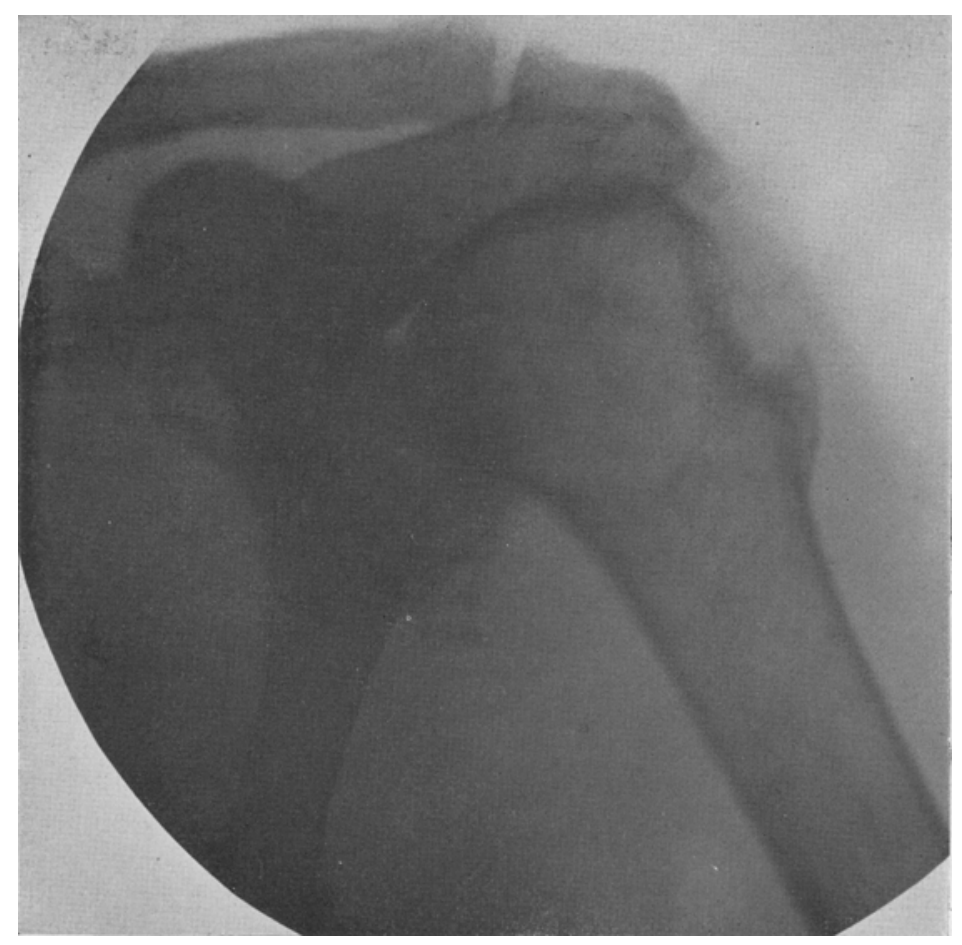

Fig. I I.

Nachuntersuchung am 5. XI. I9II: C. versieht seinen Dienst als Fuhrmann wieder in vollem Umfange; eine Unfallrente bezieht er nicht. Beim Aus- und Ankleiden benutzt er seinen im Schulter- und Ellbogengelenk versteiften Arm in geschickter Weise. Die Muskulatur der Schulter, insbesondere des Schulterblattes ist sehr, die des Armes nur wenig atrophisch; aktiv wird der rechte Arm unter geringem Krepitieren im Schultergelenk seitlich und nach vorn bis 90 Grad, nach hinten bis 55 Grad gehoben. Die Außenrotation ist fast aufgehoben, die Innenrotation nur zur Hälfte möglich. Streckung im Ellbogengelenk nur bis 150 Grad, Beugung bis 
70 Grad, Supination des Unterarmes nur zur Hälfte möglich. Geringe Verdickung der beiden IIumeruscondylen; rohe Kraft des rechten Armes kaum herabgesetzt.

Röntgenogramm (Fig. I I) zeigt starke Osteoarthritis der Komponenten des Schultergelenks, besonders der Schulterblattpfanne und eigentümlich kahnfömigen Knochendefekt in der Gegend des Tuberculum majus.

I all 9. II. A., Frau, 47 Jahre alt, aus Köln. Aufgenommen 3. VI. 1910 , entlassen 10. VI. 1910.

Diagnose: Luxatio humerisin. infraglenoidal. et fract. tuberculi maj. vetusta.

Vor 6 Wochen auf die linke Schulter gefallen, seitdem wegen „Schultergelenkskontusion" in ärztlicher Behandlung. Klagen über anfänglich heftige Schmerzen in der Schulter, die in der letzten Zeit nachgelassen haben, und Gebrauchsunfähigkeit des linken Armes.

A ufnaln mebefund: Sehr korpulente Frau mit hochgradigem Fettpolster; infolge des letzteren keine sichtbaren Veränderungen am Schultergelenk; Oberarm im Schultergelenk im Winkel ron 45 Grad seitlich abduziert, federnd fixiert und verlängert; von der Achselhöhle aus ist der Kopf, diese fast ausfüllend, zu fühlen; aktiv kann der (Oberarm nicht, passiv in sämtlichen Ebenen nur in geringem Grade bewegt werden.

Röntgenogramm zeigt Luxation des Ifumeruskopfes unter die Pfanne und Bruch des Tuberculun majus. 4. VI. In Narkose gelingt die Reposition durch Zugr am abduzierten Arme und Gegenzug an der Brust nach rechts bei gleichzeitigem Druck auf den Humeruskopf von der Achselhöhle aus. Velpeauscher Verband. Io. VI. Auf Wunsch in dic Behandlung des Ilausarztes entlassen.

Xachuntersuchung am 8. XII. IgrI: Geringe Atrophie der IIuskulatur des linken Armes: geringes Krepitieren im linken Schulterselenk; aktiv wird der linke Arm seitlich und nach vorn bis 120 Grad, nach hinten bis 60 Grad gehoben; die Außen- und Innendrehung ist nicht behindert; die rohe Kraft desselben ist nicht herabgesetzt.

F all ıo. H. S., Frau, 73 Jahre alt, aus Köln. Aufgenommen 22. III. I9II, entlassen I.3. IV. I9II.

I) iagnose: I.uxatio humeri dextr. subcoracoidea vetusta.

Vor 4 Wochen auf ebenem Boden gefallen; bisher vom IIausarzte mit Einreibungen behandelt. Klagen über völlige Versteifung des rechtert Schultergelenks und Gebrauchsunfähigkeit des rechten Armes.

A uf nahmebefund: Rechte Schulter abgeflacht; unterhalb und scitlich rom Akromion ist eine deutliche Weichteilvertiefung fühlbar; von der Achsclhöhle aus ist der llumeruskopf als dicke. 
knöcherne Prominenz fühlbar; Oberarm im Schultergelenk in einem Winkel von 45 Grad abduziert, federnd fixiert und etwas verlängert; aktiv kann er nicht, passiv kaum bewegt werden.

Röntgenogramm zeigt Luxation des Humeruskopfes vor die Pfanne und ostearthritische Veränderungen am Akromio-claviculargelenk.

24. III. In Narkose gelingt die Reposition leicht durch Zug am abduzierten Arme und Druck auf den Humeruskopf von der Achselhöhle aus; Velpeauscher Verband. - 6. IV. Verband entfernt. Bewegungstherapie. - I3. IV. Auf Wunsch zur Ambulanz entlassen; aktiv kann der Arm weder nach vorn, noch seitwärts, passiv nach vorn und seitlich bis 90 Grad gehoben werden.

Nachuntersuchung am 23. XI. I9II: Rechtes Schultergelenk, abgesehen von geringem Krepitieren (links etwas weniger vorhanden), frei beweglich; geringe Atrophie der Mm. deltoid. und supra- und infraspinat., keine der Armmuskulatur; rohe Kraft des Armes nicht vermindert.

Röntgenogramm zeigt deutliche Osteoporose und osteoarthritische Veränderungen an den Komponenten des Schultergelenks.

Wie schon erwähnt, erfolgte die unblutige Reposition in den beiden letzteren Fällen ohne besondere Mühe, was ja freilich erfahrungsgemäß auch noch für weit ältere bzw. länger bestehende Oberarmverrenkungen zutrifft; so berichtet Goya nes $\left.{ }^{1}\right)$ neuerdings über 6 Fälle aus seiner Praxis, bei denen die Luxation zwischen 15 Tagen und 2 Jahren, letztere bei einer 45 jährigen Frau, bestand; bei sämtlichen Patienten konnte er ohne irgendwelche üblen Zufälle in Narkose die Luxation unblutig und dauernd reponieren. $\mathrm{DaB}$ freilich hernach bei unseren Patienten eine mehr oder weniger große Versteifung des Schultergelenkes und damit eine nicht unbeträchtliche Beschränkung der Gebra'uchsfähigkeit des Armes eintrat, kann bei den röntgenologisch feststellbaren osteoarthitischen Veränderungen des Gelenkes weiter nicht auffällig erscheinen, zumal wenn, wie im Falle 9, außer der Verrenkung des Oberarmkopfes ein Bruch des Tuberculum majus vorhanden war. Daß gerade durch die Absprengung größerer oder kleinerer Knochensplitter, insbesondere des Tuberculum majus, auch bei sofort nach der

I) Un caso de luxación del humero muy antigua. Reduccion incruenta. Revista clin. de Madrid, Ig09, August. Ref. im Zentralbl. f. Chir. Igro. Bd. 37 . 
Cber einige seitenere Frakturen und Luxationen des Oberaminopies usw. 509

Verletzung vorgenommenen Reposition das funktionelle Resultat in hohem Maße gefährdet wird, darauf hat auch $G$ öbel') auf Grund des Nachuntersuchungsergebnisses einer größeren Anzahl von Patienten des Kölner Bürgerhospitals mit vollem Rechte hingewiesen.

I) Zur l'rognose der traumatischen Schultergelenksluxation. Zeitschr. f. ärti. liurtbilcung Igog, Jairg. VI. 